\title{
ESTIMACIÓN DE LA EROSIÓN HÍDRICA PARA EL USO ACTUAL Y EROSIÓN POTENCIAL DEL SUELO EN LA FINCA AGRÍCOLA EXPERIMENTAL UNI, MUNICIPIO “LAS FLORES", DEPARTAMENTO DE MASAYA
}

\section{ESTIMATION OF WATER EROSION FOR THE CURRENT USE AND POTENTIAL EROSION OF THE SOIL IN THE EXPERIMENTAL UNI AGRICULTURAL FARM, “LAS FLORES” MUNICIPALITY, MASAYA DEPARTMEN}

\author{
Rosario Soleto Contreras ${ }^{1}$ \\ Oscar Salvador Abaunza Pérez $z^{2}$ \\ Katherine Esperanza García Montoya ${ }^{3}$ \\ Miguel Enrique Blanco Chávez ${ }^{4}$
}

(recibido/received: 3-septiembre-2021; aceptado/accepted:24-noviembre-2021)

RESUMEN: En el presente artículo se aborda el estudio Estimación de la erosión hídrica para el uso actual y erosión potencial del suelo en la finca experimental UNI utilizando la Ecuación revisada de la ecuación Universal de Pérdida de Suelo (RUSLE). El objetivo fue estimar la erosión hídrica para el uso actual y erosión potencial del suelo en la Finca Experimental de la UNI. La Finca tiene una extensión de 49.50 manzanas, utilizada para la realización de prácticas y con fines investigativos por parte de los estudiantes de la carrera de ingeniería agrícola. Se estimó la erosión hídrica superficial mediante el uso de la Ecuación Universal de Pérdida de Suelo Revisada (RUSLE). Para utilizar este modelo se obtuvieron los factores de erosividad, erodabilidad, la longitud y el gradiente de la pendiente, cobertura y prácticas de conservación. Para ello se realizaron una serie de actividades entre ellos la recopilación de datos de campos como el uso actual del suelo, topografía de la zona, precipitaciones diarias y mensuales y los respectivos análisis para la determinación de las propiedades físicas y químicas del suelo. El factor de erodabilidad $(K)$ teórico se obtuvo a través de cuatro propiedades del suelo (Textura, estructura, materia orgánica y permeabilidad) dichos valores fueron introducidos en el nomograma de Wischmeier (1971). El estudio sugiere que en la Finca Agrícola Experimental de la Universidad Nacional de Ingeniería (FAE-UNI) se provoca tasas de erosión hídrica superficial menores que 12 Ton/ (ha-año).

\footnotetext{
${ }^{1}$ Facultad de la Tecnología de la Construcción. Universidad Nacional de Ingeniería. Managua, Nicaragua. correo: rosario.sotelo@ftc.uni.edu.ni

2 Ingeniero Agrícola. Universidad Nacional de Ingeniería. Managua, Nicaragua.correo: oabaunzaperez@gmail.com

3 Ingeniero Agrícola Universidad Nacional de Ingeniería. Managua, Nicaragua, correo: Katherine.montoya150399@gmail.com

4 Programa de Investigación, Estudios Nacionales y Servicios del ambiente correo: miguel.blanco.@piensa.uni.edu.ni
} 
PALABRAS CLAVES: Erosividad, Erodabilidad, Lluvia, Erosión hídrica.

ABSTRACT: This article addresses the study Estimation of hydric erosion for the current and potential erosion of the soil in the UNI experimental farm using the RUSLE method. The objective was to estimate the hydric erosion for the current and potential erosion of the soil in the Experimental Farm of the UNI. The farm has an area of 49.50 blocks, used for agricultural activities. Surface water erosion was estimated using the Revised Universal Soil Loss Equation (RUSLE). To use this model the factors of erosivity, erodability, length and gradient of the slope, coverage and conservation practices were obtained. For this, a series of activities were carried out, including the collection of data from fields such as current land use, topography of the area, daily and monthly rainfall and the respective analyzes to determine the physical and chemical properties of the soil. The theoretical erodibility factor $(K)$ was obtained through four soil properties (Texture, structure, organic matter and permeability), these values were introduced in the Wischmeier (1971) nomogram. The study suggests that the FAE-UNI causes superficial water erosion rates lower than 12 Ton / (ha-year).

KEYWORDS: Erosivity, Erodability, Rainfall, Hydric erosion.

\section{INTRODUCCIÓN}

La Universidad Nacional de Ingeniería (UNI) actualmente cuenta con la Finca Agrícola Experimental la cual utilizará la abreviatura FAE-UNI para fines didácticos e investigaciones agrícolas, se han establecido cultivos como plátano, yuca, pipián y árboles frutales, entre otros, siendo producto de las prácticas que realizan los estudiantes de la carrera de ingeniería agrícola. Los suelos se caracterizan por tener textura franco arcillosa limosa y con un terreno de poca pendiente, sin embargo, existe erosión lo que causara graves daños a través del tiempo.

En el presente estudio se realizó la estimación de la erosión hídrica superficial en el uso actual y erosión potencial de los suelos de la Finca Agrícola Experimental de la UNI, ubicada en el Municipio "Las Flores", Departamento de Masaya. Utilizando el método Ecuación Universal de Pérdida de Suelo propuesta por (Wischmeier \& Smith, 1976) mediante las ecuaciones de estimaciones empíricas. Para aplicarse el método RUSLE (Ecuación modificada) se recopilaron datos existentes los cuales son, uso del suelo, tipo de suelo, permeabilidad, topografía de la zona de estudio en formato digital, imágenes satelitales, prácticas de cultivos, precipitaciones diarias, mensuales y anuales, intensidades máximas anuales, cartas pluviográficas y los respectivos análisis del suelo.

Se realizaron visitas de campo a la finca, se levantó información del uso de suelo, de prácticas de cultivos y conservación de suelos. La información recopilada y analizada se utilizó en la actualización de mapas temáticos en formato digital, como mapas geoespaciales haciendo uso del QGIS. 


\section{METODOLOGIA}

Estimación de la erosión hídrica superficial del suelo

En base al análisis estadístico de los datos experimentales los investigadores Wischmeier \& Smith (1971, pp. 3-4), en una publicación del Departamento de Agricultura de EUA presentan la Ecuación Universal de Pérdida de Suelo (USLE), que permite estimar la perdida promedio anual de suelo para un sitio específico bajo condiciones particulares de cultivos, tipo de suelo, pendiente y prácticas de cultivos. En la ecuación 1 se describe los factores que interviene para estimar las pérdidas de suelos por erosión hídrica.

$$
A=\text { R K L S C P }
$$

Donde:

$A$ = Es la pérdida de suelos calculada por unidad de superficie, expresada en las unidades seleccionadas para $\mathrm{K}$ y el período seleccionado para $\mathrm{R}$, generalmente toneladas por hectárea por año, (Ton/ha-año).

$\mathrm{R}=$ El factor lluvia y escurrimiento o índice de erosividad de la lluvia, es el número de unidades de índice de erosión pluvial (EI), en (MJ/ha - mm/h). El índice de erosión pluvial (EI) para una tormenta es el producto de la energía total de la tormenta (E) y su máxima intensidad en 30 minutos (I).

$\mathrm{K}=$ El factor susceptibilidad de erosión del suelo o erodabilidad del suelo, es la tasa de pérdida de suelos por unidad $\mathrm{E}^{\star} \mid$ para un suelo específico, medido en una parcela experimental o terreno estándar (22.13 m de largo, 9\% pendiente, en barbecho y labranza continua).

$\mathrm{L}=\mathrm{El}$ factor de largo de la pendiente, es la proporción de pérdida de suelos con una longitud pendiente especifica con respecto a la pérdida de suelo con una longitud de pendiente estándar $(22.13 \mathrm{~m})$. Este factor es adimensional.

$\mathrm{S}=\mathrm{El}$ factor de magnitud de la pendiente, es la proporción de pérdida de suelos de una superficie con una pendiente especifica con respecto a la pérdida de suelo en la pendiente estándar de $9 \%$, con todos los otros factores idénticos. Este factor es adimensional.

$\mathrm{C}=\mathrm{El}$ factor cubertura y manejo, es la proporción de pérdida de suelo en una superficie con cubierta y manejo especifico con respecto a una pérdida de suelo en superficie idéntica en barbecho, con labranza continua. Este factor es adimensional.

$\mathrm{P}=\mathrm{El}$ factor de prácticas de conservación, es la proporción de pérdida de suelo con una práctica de apoyo como cultivo en contorno, barreras vivas, o cultivo en terrazas, con respecto a aquella labranza en el sentido de la pendiente. Este factor es adimensional. 
Este método fue ligeramente modificado por (Renard et al, 1997) del Departamento de Agricultura de EUA, llamándose Ecuación Universal Revisada de Pérdida de Suelos (RUSLE).

(Renard et al, 1997) afirman que "RUSLE es un modelo de erosión diseñado para predecir la pérdida de suelo promedio anual (A) a largo plazo, provocada por la escorrentía en un lugar específico con sistemas de manejo y cultivos específicos. Su amplio uso ha confirmado la utilidad y validez del RUSLE para este propósito. Este también es aplicable en condiciones no agrícolas tales como sitios de construcción" (Wischmeier \& Smith, 1971, pág. 15).

\section{Simulador de lluvia}

Para efecto de obtener datos para calcular $\mathrm{K}$ experimental, se utilizó un simulador de lluvia, con el cual es posible cuantificar la escorrentía, infiltración y pérdida de suelo generados por una regadera de lluvia ajustable a la intensidad sobre un área de prueba definida (Monge R \& Aguilar J, 2008).En la figura 1 se muestra el esquema del equipo.

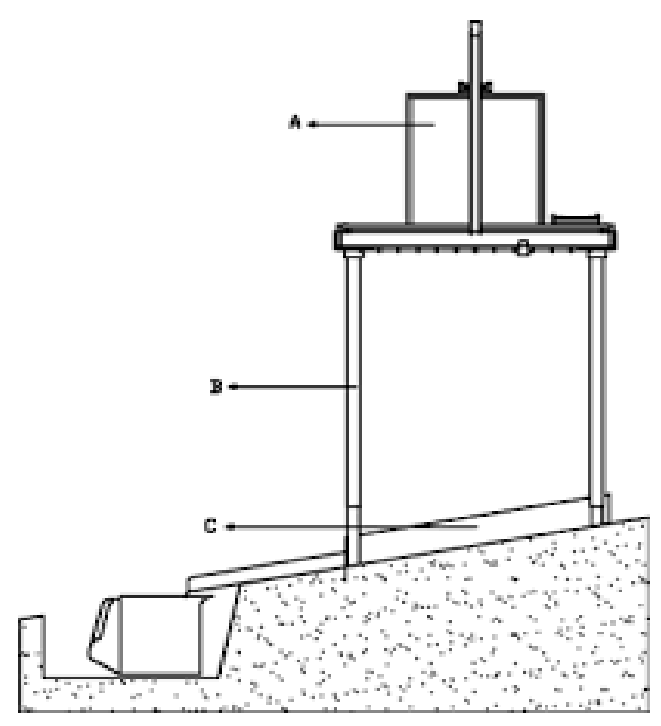

Figura 1: Representación del simulador de lluvia

Fuente: (Monge R \& Aguilar J, 2008)

El estudio se realizó en la Finca Experimental Agrícola, ubicada en el departamento de Masaya, región Centro - Pacífico de Nicaragua, con $610.78 \mathrm{Km}^{2}$ de superficie. El departamento de Masaya está ubicado en el kilómetro 31 de la carretera Masaya, del empalme Las Flores 8 kilómetros al este, sobre el camino de la calle principal que conecta la comarca La Bolsa con la rotonda Las Flores entre las coordenadas N1200'5.33" W8559'90.8”, figura 2. 


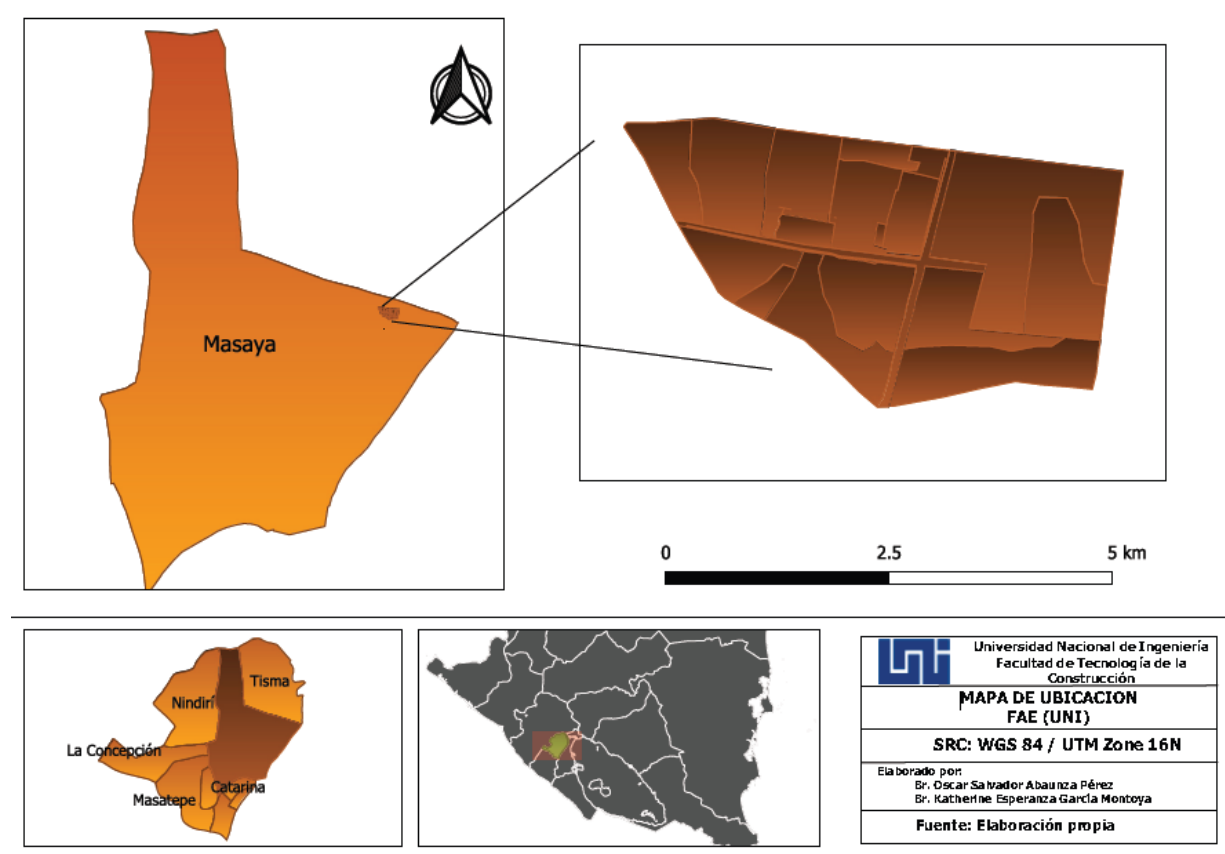

Figura 2: Localización de la zona de estudio

Descripción fisiográfica: La Comunidad "Santa Clara" está cerca de la "Provincia de la Depresión de Nicaragua, Sub provincia planicies de Tipitapa" que comprende las llanuras de relieve bajo, constituida en la superficie por depósitos sedimentarios reciente. El drenaje superficial escurre hacia el Río Tipitapa, laguna de Tisma y Río Malacatoya para desembocar luego en el lago de Nicaragua. (Cerrato L \& Téllez E, 2006)

Factores de erosión:

La determinación del factor K:

- Factor K (teórico) por el método del nomograma

La estructura del suelo se determinó mediante una calicata realizada en las coordenadas $\mathrm{X}$ : 608747.3 Y: 1328154.94, para luego hacer énfasis en la estructura obtenida por medio del código de estructura del suelo.

Para el cálculo de $\mathrm{K}$ teórico fueron necesarios los valores correspondientes de Textura, La materia orgánica (m.o), La estructura y la permeabilidad, parámetros que tienen un código dependiendo del grado y tamaño de la muestra y de la textura del suelo respectivamente.

Para encontrar los valores en el nomograma de Wischmeier, ver figura 3, se inició la lectura de izquierda a derecha iniciando en la gráfica (a) y terminando en la gráfica (b). En primer lugar, se ubicó el valor del porcentaje de textura de partículas de $0.002-0.10 \mathrm{~mm}$ resultando (95.6) en el eje $Y$ (ordenada) de la gráfica (a), trazando una línea en el sentido antes explicado e interceptando la zona correspondiente al porcentaje de arena (4.4), siguiendo con el proceso se trazó una línea hacia arriba interceptando la zona correspondiente al porcentaje de M.O (4.27) para luego trazar una línea 
directamente a la gráfica (b) interceptando la zona correspondiente a la estructura del suelo, luego se trazó una línea hacia abajo interceptando la zona correspondiente a la permeabilidad. Para finalizar se trazó una línea hacia la izquierda interceptando el eje $\mathrm{Y}$, y dando como resultado el valor K inglés (0.44).

Una vez obtenido estos valores se ubicaron en el nomograma dando como resultado el factor $\mathrm{K}$ en unidades inglesas, por lo que posterior a su selección se divide entre 7.59 (Blanco M, 2019) que es un factor de conversión, para llevarlo a unidades del sistema internacional. En el presente estudio se calculó un solo valor de $\mathrm{K}$ en toda la finca experimental agrícola por lo que el $\mathrm{K}$ en cada polígono es el mismo.

\section{- Factor K experimental}

Del ensayo con el simulador de lluvia mediante previos aforos, se obtuvo el volumen aplicado y recolectado, así como los sedimentos húmedos. En el laboratorio se pesaron los sedimentos húmedos para ingresarlos en el horno por 24 horas, obteniendo así el peso de estos en seco, la suma de ambos pesos son los sedimentos totales erosionados en gramos.

El volumen de agua aplicado se obtuvo en litros, mientras que el volumen recolectado en mililitros, realizando la conversión de estas unidades a metros y milímetros respectivamente, el tiempo de ensayo se tomó en minutos y se convirtió a horas, los sedimentos totales a toneladas y el área de ensayo a hectáreas.

\section{Intensidad de la lluvia}

Una vez que se obtuvieron los datos del ensayo de la simulación de lluvia en las unidades requeridas se procedió al cálculo de la intensidad de lluvia aplicada y de la infiltración. A continuación, se presenta en la ecuación 2 la fórmula de la intensidad de lluvia.

$I=\frac{h_{\text {sim }}}{t_{\text {apli }}}$

\section{Donde}

I: Intensidad de lluvia aplicada $\left(\frac{\mathrm{mm}}{\mathrm{hr}}\right)$

$h_{\text {sim }}=$ Altura de lluvia aplicada $(\mathrm{mm})$

$\mathrm{t}_{\mathrm{apli}}=$ Tiempo de ensayo $(\mathrm{hr})$

Por último, la infiltración se obtuvo restando el volumen de lluvia aplicado al volumen recolectado. Los parámetros utilizados para la determinación del Factor K experimental, fue mediante IK, utilizado por (Murillo G, 1990),detallándose en la ecuación 3 siguiente el índice de k experimental.

$$
I K=\frac{A}{R \cdot L \cdot S}
$$


Donde:

Ik: Índice k experimental (Ton/ha) / (MJ/ha-mm/hr)

A: Erosión (ton/ha)

$\mathrm{R}$ : Erosividad de la lluvia (MJ/ha-mm/hr)

L: Factor de la longitud de la pendiente

S: Factor de la pendiente

A continuación, se muestran las ecuaciones utilizadas para cada uno de los parámetros utilizados para calcular IK:

A continuación, se presenta las pérdidas de suelo (A), ecuación 4.

- $A=\frac{g t}{A_{\text {ensayo }}}$

Donde:

gt $=$ Sedimentos totales erosionados (ton)

$\mathrm{A}_{\text {ensayo }}=$ Área del ensayo (ha)

Ecuación 5 tiene como finalidad encontrar la erosividad de la lluvia.

- $\quad \boldsymbol{R}=\boldsymbol{E} * \boldsymbol{I}_{\mathbf{3 0}}$

Donde: $E=\mathrm{e}^{*} \mathrm{~h}$

$\mathrm{e}=0.1189+0.0873 \log 10$ (I)valida para I $<=75 \frac{\mathrm{mm}}{\mathrm{h}}$, cuando I $>75 \mathrm{~mm} / \mathrm{h} \mathrm{e}=0.283$

$h=$ Altura de la lluvia aplicada

$I_{30}=$ Intensidad de la lluvia aplicada

Se muestra a continuación (ecuación 6) el factor de longitud de la pendiente de (Foster et al, 1977):

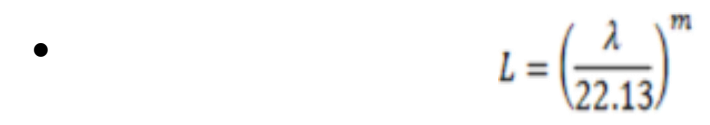

Donde:

$L=$ factor longitud de la pendiente, adimensional.

$\mathrm{l}=$ longitud del área en estudio $(\mathrm{m})$

$\mathrm{m}=$ exponente, al simular una pendiente de $3 \%$, se utilizó un $\mathrm{m}=0.4$

También se toma en cuenta la inclinación de la pendiente adimensional, ecuación 7.

- $S=10.8 \operatorname{Sen} \theta+0.03$ 
Factor de erodabilidad K utilizada por (Murillo G, 1990, págs. 31-32), ecuación 8.

$\mathrm{K}=200.6 \mathrm{IK}-0.038(8)$

La determinación del factor de erosividad R:

Donde:

$E I_{30}=$ Índice de erosividad de un evento lluvioso erosivo.

El valor de E se calcula en base de las diferentes intensidades de los intervalos de una lluvia. La determinación del El_30de una tormenta es:

E=Energía Cinética total para un evento de precipitación

$E I_{30}=$ Intensidad máxima de la precipitación en 30 minutos.

La energía cinética por unidad de precipitación (e) por milímetros de precipitación es igual a (ecuación 9 y 10):

$e=0.1189+0.0873 \log _{10} I$

Donde:
$\mathrm{l}=$ Intensidad del $=\sum \boldsymbol{E I}_{\mathbf{3 0}}$ intervalo de precipitación $(\mathrm{mm} / \mathrm{h})(10)$

E es calculada como la sumatoria de los productos de la energía cinética por unidad de lámina de lluvia (e) y la intensidad de precipitación (la) del intervalo considerado.

$E=\sum e I a$

Donde:

$E I_{30}$ Se determina analizando la distribución de las intensidades en una lluvia y seleccionando los treinta minutos de duración que posean la máxima intensidad. Si la lluvia es de menos de 30 minutos de duración, se utiliza entonces la intensidad total de dicho periodo.

El índice modificado de Fournier es lo más práctico en zonas donde no existen datos detallados de intensidades. Como base para determinar la erosividad se utilizan los datos promedios de precipitaciones mensuales y el mapa de isoyetas. Este índice se obtiene mediante la siguiente fórmula (ecuación 11):

$I M F=\sum_{i=1}^{i=12} \frac{p i^{2}}{P}$ 
Donde:

i= Representa el número del mes, pi $=$ precipitación mensual $(\mathrm{mm})$

$\mathrm{P}=$ Precipitación promedio anual $(\mathrm{mm})$

La erosividad se puede medir por mes, por periodos al año o por todo el año. (INETER \& COSUDE, 2005, págs. 41-42)

\section{- Factor erosividad de la lluvia $R$}

Para calcular el factor $\mathrm{R}$, se recopilaron datos de precipitación mensual de las estaciones meteorológicas de INETER ubicadas en Masaya, Granada y Panaloya, las últimas dos se utilizaron por su cercanía a la zona en estudio.

Las estaciones de Granada y Panaloya no cuentan con registros de precipitación completa, por lo que se procedió a completarlos mediante la ecuación correlación lineal (ecuación 12 y 13). Esta ecuación mencionada se obtuvo realizando una gráfica con los datos de precipitación mensual de las estaciones de los diez años en estudio, para esta grafica se agruparon los datos de la estación de Masaya-Granada, Masaya-Panaloya, puesto que la estación de Masaya es la única que cuenta con los datos de precipitación anual de los diez años.

Resultando:

Masaya-Granada: $y=0.8501 x+27.539(12)$

$$
r^{2}=0.7275
$$

Masaya-Panaloya: $y=0.6643 x-2.966(13)$

$$
r^{2}=0.7476
$$

Siendo $\mathrm{X}$ el valor correspondiente para cada precipitación mensual de la estación de Masaya y $\mathrm{r}^{2}$ que indica el nivel de confianza del uso de estas ecuaciones, esto refiere a que $\mathrm{r}^{2}$ sea aceptable sí se encuentra dentro del rango de $0.5-1$, preferiblemente que sea más cerca de 1 . NOTA: ${ }^{2}$ se llama coeficiente de determinación.

Una vez completados los datos de precipitación de las estaciones se procedió a calcular el índice modificado de Fournier (IMF) que corresponde a la ecuación 14 mostrada a continuación.

$$
\mathrm{IMF}=\sum_{i=1}^{i=12} \frac{p i^{2}}{P}
$$


La fórmula permite estimar $\mathrm{R}$ como factor de erosión. Posteriormente se aplicó el índice modificado de Fournier (IMF) para los diez años en estudio.

Finalmente, para el cálculo de $\mathrm{R}$ de las tres estaciones meteorológica se utilizó la ecuación $R=29.81 I M F-375.9$ referida por (Blanco M, Erosión hídrica en la microcuenca del cauce 31 de diciembre, 2019), realizándose un promedio total para cada estación y por último se promedió el $R$ de las tres estaciones.

- Factor longitud de la pendiente $\mathrm{L}$

Una vez realizadas las visitas de campo a la finca, usando el software QGIS se trazaron diferentes polígonos para cada área y cultivos de la finca, esto con el fin de obtener la longitud los polígonos necesarios para el cálculo de L.

La ecuación 15 del cálculo del factor longitud de pendiente es:

$L=\left(\frac{\lambda}{22.13}\right)^{m}$

Donde:

$\lambda=$ longitud de la pendiente del tramo en estudio $(\mathrm{m})$

$\mathrm{L}=$ factor longitud de la pendiente, adimensional.

$\mathrm{m}$ = exponente que refleja la relación entre la pérdida de suelo y el gradiente de la pendiente, este valor ya está indicado en tablas elaboradas en (INETER \& COSUDE, 2005). Tabla 1.

Tabla 1: Valores del exponente $(\mathrm{m})$ según la pendiente del suelo.

\begin{tabular}{|c|c|}
\hline $\begin{array}{c}\text { Exponente } \\
(\mathrm{m})\end{array}$ & Pendiente del suelo en \% \\
\hline 0.2 & $<1$ \\
\hline 0.3 & $1-3$ \\
\hline 0.4 & $3-5$ \\
\hline 0.5 & $5-10$ \\
\hline 0.6 & $10-50$ \\
\hline
\end{tabular}

Fuente: (INETER \& COSUDE, 2005)

- Factor inclinación de la pendiente S

Para calcular el factor $S$ se trazaron polígonos en la finca, los que resultaron ser pequeños en comparación a otras áreas estudiadas, como son las cuencas e incluso microcuencas, en este caso no fue necesario generar la capa ráster del DEM generada en QGIS, la cual permite obtener información espacial del área de estudio. 
En cambio, se utilizaron la siguiente ecuación 16, dependiendo del porcentaje de pendiente de cada polígono que corresponde al factor de inclinación de la pendiente.

$S=10.8 \operatorname{Sen} \theta+0.03 \quad \mathrm{p}<9 \%$

Donde:

$\mathrm{S}=$ factor inclinación de la pendiente (adimensional)

$\Theta=$ ángulo de la pendiente del suelo (grados)

$\mathrm{p}=$ pendiente del suelo $(\%)$

- Factor cobertura vegetal del suelo C

Al no existir valores de C universalmente válidos por la gran variedad de cultivos, secuencias, rotaciones de cultivos y manejos, primeramente, se determinó el uso actual del suelo de cada polígono y según el tipo de cobertura vegetal existente se asignaron los distintos valores del factor vegetación, utilizando valores ya establecidas por diversas fuentes, donde presentan el valor de $\mathrm{C}$ en función del tipo de cultivo.

- Factor prácticas de conservación $P$

Debido a que en la finca agrícola experimental no se han realizado prácticas de conservación de suelos al momento de establecer los cultivos agrícolas ni posterior a ellos, se asumió un valor de $(P=1)$.

\section{RESULTADOS Y DISCUSIÓN}

Determinación de las propiedades físicas y químicas

Para la determinación de estas propiedades se seleccionaron las diferentes áreas cultivadas actualmente. El suelo presenta una condición porosa alta, favorable para el crecimiento de las raíces, buena aireación y una infiltración alta del agua (función hidrología del suelo). Tomando en cuenta los tamaños de partículas de suelo en prueba de campo, se determinó una superficie áspera y al apretarlo entre los dedos es pegajoso, granuloso y mancha los dedos (para suelos franco arcillosos limosos). En la siguiente tabla 2 se encuentran los resultados de cada propiedad.

Tabla 2: Resumen de los resultados de las propiedades de suelo en la FAE-UNI.

\begin{tabular}{cccc}
\hline Propiedades & \multicolumn{2}{c}{ Resultados } & Clasificación \\
\hline & $\mathrm{N}^{\circ} 4$ & 0.76 & Muy baja \\
& $\mathrm{R} . \mathrm{L}$ & 0.75 & Muy baja \\
& $\mathrm{B}$ & 0.88 & Muy baja \\
$\mathrm{Da}\left(\mathrm{gr} / \mathrm{cm}^{3}\right)$ & $\mathrm{SRS}$ & 0.78 & Muy baja \\
& $\mathrm{B} 3$ & 0.98 & Muy baja \\
& $\mathrm{B} 1$ & 0.76 & Muy baja \\
$\mathrm{Dr}\left(\mathrm{gr} / \mathrm{cm}^{3}\right)$ & $\mathrm{N}^{\circ} 6$ & 2.14 & Baja \\
\hline
\end{tabular}

El Higo Revista Científica / Volumen 11. No. 02, pp. 28-47/ diciembre 2021 
Rosario V. Sotelo C.; Oscar S. Abaunza. P.; Katherine E. García E.; Miguel E. Blanco

\begin{tabular}{cccc}
\hline & $\mathrm{N}^{\circ} 4$ & 16.08 & AGR \\
& R.L & 12.59 & AGR \\
& B & 13.81 & AGR \\
Humedad (\%) & SRS & 9.36 & AGR \\
& B3 & 12.07 & AGR \\
& B1 & 15.25 & AGR \\
Textura & & Arcilla: & \\
& & $32.48 \%$ & Franco arcillosa \\
& S.NO1 & Limo: & limosa \\
& & $63.12 \%$ & \\
& & Arena: $4.4 \%$ & \\
& & 64.35 & Alta \\
Porosidad (\%) & & 64.92 & Alta \\
& & 58.74 & Alta \\
& & 64.74 & Alta \\
C.C (\%) & No 6 & 64.76 & Mediana \\
M.O (\%) & & 4.27 & Alta \\
& & & Mediana \\
\hline
\end{tabular}

\section{Factor K teórico y experimental}

Una vez obtenido los datos en el nomograma, se encontró $\mathrm{K}$ en unidades del SI (Sistema Internacional) siendo su unidad de medida (Ton/ha-año) /(MJ-mm/ha-h), para esto el valor de 0.44 se dividió por 7.59 (Blanco M, 2019), obteniendo un resultado del k teórico o K de gráfico: 0.06.

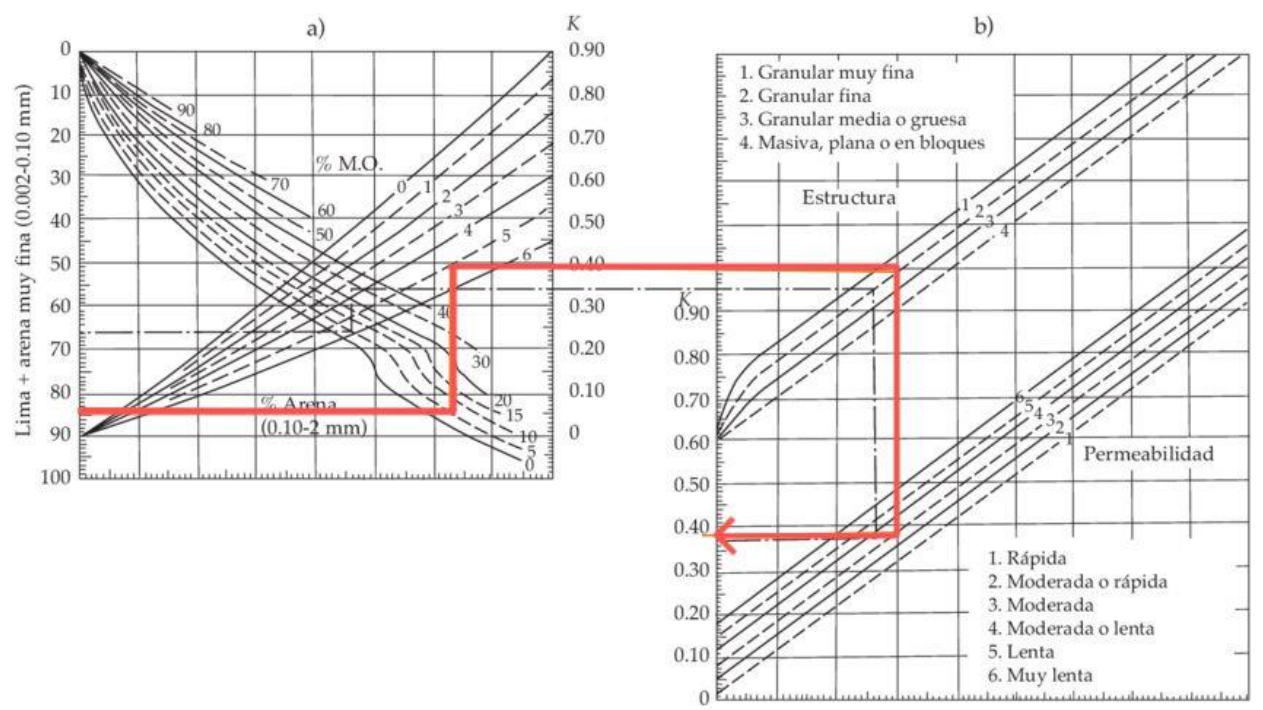

Figura 3: Resultado del nomograma 


\section{Kexperimental}

El K experimental encontrado en la FAE mediante la prueba con el simulador de lluvia donde se midió el volumen de agua en un tiempo determinado y así mismo los sedimentos que resultaron en el tiempo del aforo se obtuvieron los resultados mostrados en la tabla 3.

Tabla 3: Factores para encontrar K (erodabilidad) experimental

\begin{tabular}{lll}
\hline Factor & Unidad & Valor \\
\hline $\mathrm{A}$ & Ton/ha & 9 \\
$\mathrm{R}$ & (MJ/ha-mm/hr) & 287564 \\
$\mathrm{~L}$ & Adimensional & 0.205 \\
$\mathrm{~S}$ & Adimensional & 0.354 \\
$\mathrm{I}$ & $\mathrm{mm} / \mathrm{hr}$ & 3810 \\
$\mathrm{E}$ & $\mathrm{MJ} / \mathrm{ha}$ & 75.48 \\
$\mathrm{E}$ & $(\mathrm{MJ} / \mathrm{ha}-\mathrm{mm}))$ & 0.283 \\
$\mathrm{H}$ & $\mathrm{Mm}$ & 266.7 \\
$\mathrm{Gt}$ & Ton & 0.00016 \\
$\mathrm{IK}$ & $($ Ton/ha $) \quad / \quad(\mathrm{MJ} / \mathrm{ha}$ & $-4.32050 \mathrm{E}-$ \\
& $\mathrm{mm} / \mathrm{h})$ & 04 \\
$\mathrm{~K}$ & $(\mathrm{Ton} / \mathrm{ha}) \quad / \quad(\mathrm{MJ} / \mathrm{ha}$ & -0.05 \\
& $\mathrm{~mm} / \mathrm{h})$ & \\
\hline
\end{tabular}

La erodabilidad del suelo por medio de K teórico utilizando el nomograma de (Wischmeier \& Smith, 1971), arrojó un valor 0.06, calificados como bajo, es decir resistente al desprendimiento por la salpicadura o flujo laminar. Mientras que EI K experimental resulto ser de 0.05 clasificado como rango bajo, coincidiendo con el valor de $\mathrm{K}$ teórico, en que ambos valores indican que el suelo presenta resistencia al desprendimiento por efecto de la lluvia.

\section{Factor erosividad de la lluvia $R$}

Una vez completada las tablas de precipitación se estimaron los valores de precipitación histórica anual para cada estación, resultando para la estación de Masaya 1366 mm, Granada 1545 mm y Panaloya $791 \mathrm{~mm}$, ocurriendo la mayor actividad de lluvias entre los meses de Mayo a Octubre.

Los valores de R en MJ.mm. $h a^{-1}$ obtenido mediante el IMF fueron 7264.8, 7158.3, 4922.4 para la estación de Masaya, Granada y Panaloya respectivamente. Teniendo en cuenta los registros históricos de las tres estaciones se pueden clasificar como moderadamente lluvioso, ya que están en el rango entre 500 y 2000 mm. Existe una relación entre los valores de precipitación y el resultado del factor $\mathrm{R}$, es decir, que sí las precipitaciones son altas $\mathrm{R}$ también lo será y sí las precipitaciones son bajas de igual manera lo será $\mathrm{R}$. Como se puede apreciar los valores de R están coherentes a los datos de las precipitaciones anuales clasificándose para Masaya y Granada como moderadas, 
Rosario V. Sotelo C.; Oscar S. Abaunza. P.; Katherine E. García E.; Miguel E. Blanco

Panaloya al contar con el menor registro de precipitación su $\mathrm{R}$ es el menor de las tres estaciones, clasificándose como baja.

Factor de longitud de la pendiente L y $S$

El área de estudio tiene pendientes que van entre el rango de 0.1 a $0.3 \%$, los cálculos de LS se determinaron con la fórmula establecida por (Wischmeier \& Smith, 1976) y se tomó como referencia la pendiente menor a $9 \%$. Aunque la pendiente resulto menor de 1\%, dichas pérdidas están relacionadas a otros factores, como pendientes menores que influencian la velocidad de escurrimiento del agua de manera lenta, provocando en el tiempo desprendimiento de las partículas expuesta en la capa superficial. Así mismo sucede con el factor longitud (L), al existir distancias cortas la influencia en la escorrentía provoca lento movimiento de las partículas a través del flujo laminar que es insidioso en el tiempo, pasando muchas veces desapercibido, pero generando lavados. Los porcentajes de pendiente obtenidos en la FAE están clasificados como pendientes suaves, no aumentan la velocidad de flujo superficial y por ende no provoca un desprendimiento severo del suelo.

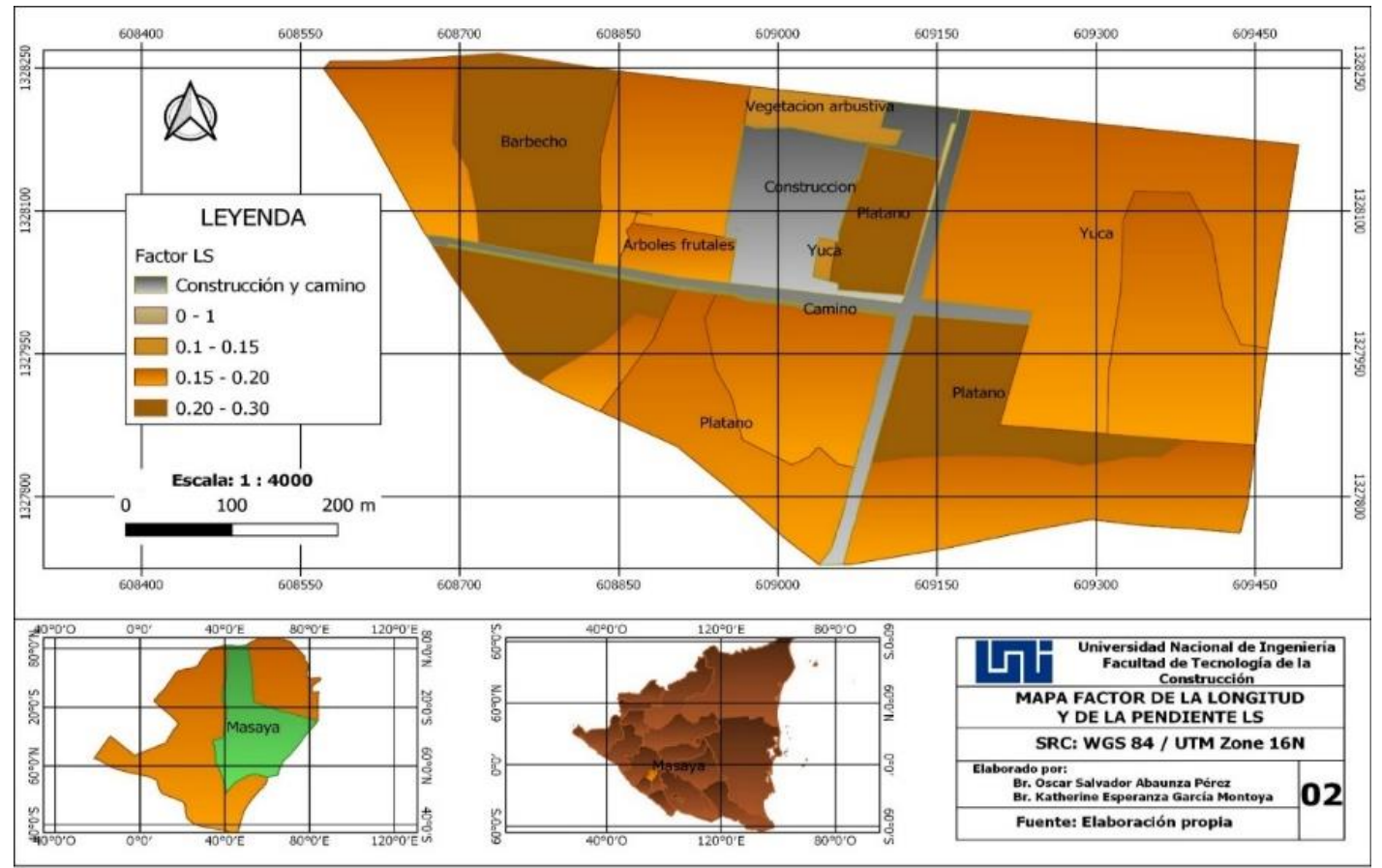

Figura 4: Mapa factor de la longitud $L$ y de la pendiente $S$

\section{Factor de cobertura $C$}

El valor de factor $\mathrm{C}$ del cultivo yuca (Manihot esculenta) fue de 0.20 , dato que resultó ser mayor en relación a los demás cultivos presentes, debido a las características morfológicas de la planta que influencia la cubierta foliar y que deja más desprotegido el suelo frente por la lluvia. El impacto de la lluvia es más directo por el menor porcentaje de área foliar disminuyendo la protección, por lo tanto, donde está establecido la yuca en la finca, existe una mayor erosión en comparación a los demás sitios. 
En cambio, donde se encuentran otros cultivos, como es el caso del cultivo de plátano con un valor de 0.08 , estos valores de erosión son menores debido a la influencia que ejerce la cobertura foliar que absorbe la energía de las gotas de lluvia y disminuye la velocidad de escurrimiento. Se puede observar que los barbechos tienen valores bajos de 0.01 , lo que significa más protección a los suelos.

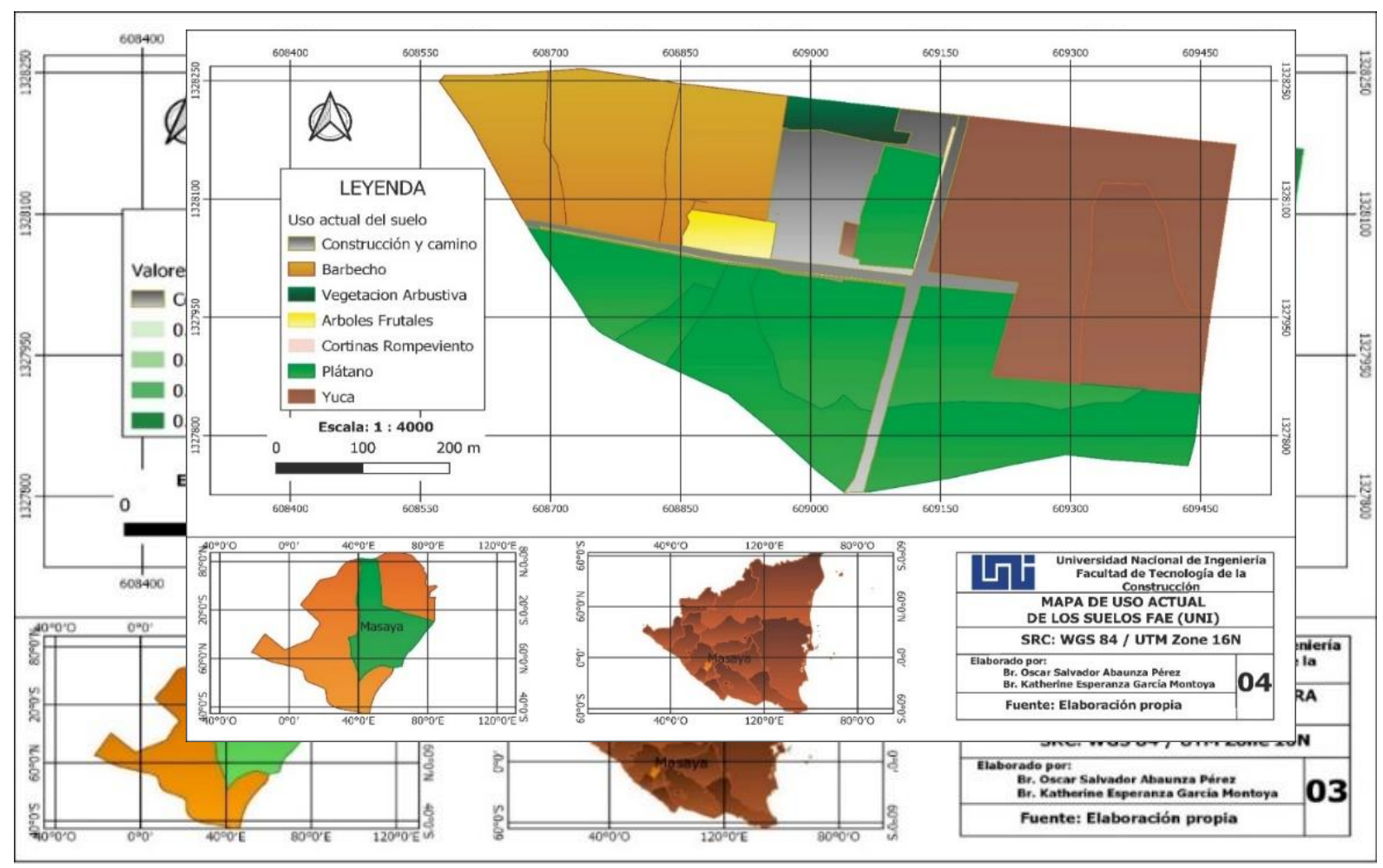

Figura 5: Mapa factor de la cobertura vegetal C.

\section{Factor prácticas de conservación $P$}

En el área de estudio, no se identificaron prácticas de conservación de suelo para la prevención o control de la erosión hídrica por lo que se le asignó a $\mathrm{P}$ el valor 1.

Uso actual de los suelos de la FAE

En el uso actual de los suelos se presenta en la figura 6, muestra el mapa con los diferentes cultivos presentes en la finca experimental UNI, siendo el cultivo de plátano el más extenso abarcando 12.9 ha, la yuca con 9.3 ha, barbecho con 6.3 ha, un área de vegetación arbustiva con 0.6 ha, árboles frutales con 0.5 ha, cortinas rompevientos con 0.1. ha. (Espinales E. CATASTRO, 1971) Citado por (Rivera C \& Alguera E, 2016) coinciden que los problemas de conservación de estos suelos son calificados de ligeros a moderadamente ligeros y se requieren prácticas de conservación tales como: cultivos en contornos, mínimas labores de labranzas, y rotación de cultivos. 


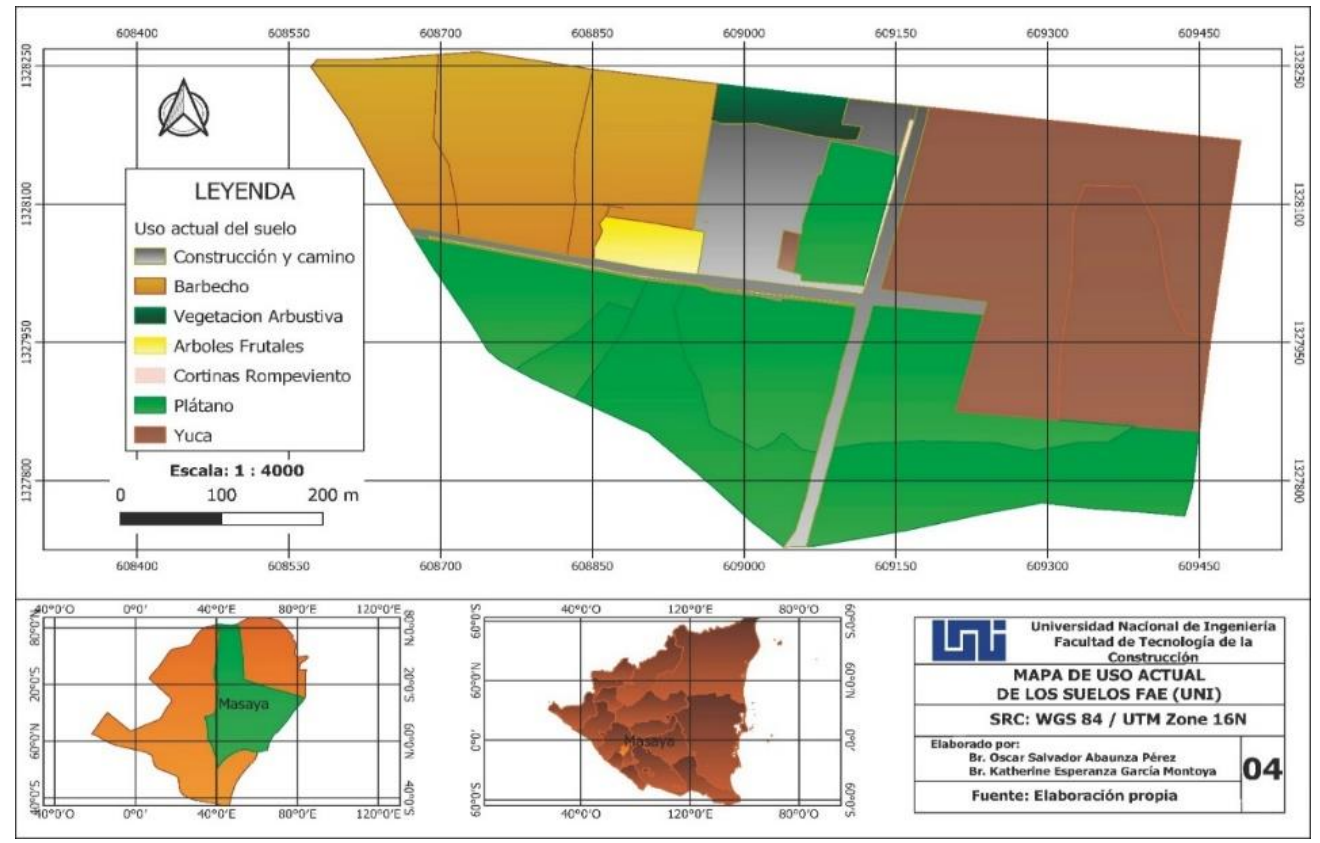

Figura 6: Mapa de uso actual de los suelos FAE (UNI)

\section{Erosión hídrica actual de los suelos}

La pérdida actual del suelo se estimó al considerar el producto de los factores (R, K, LS, C, P) que conforman la RUSLE; se generó el mapa de la erosión actual, figura 7 , observando que solo el $27 \%$ del territorio se encuentra con el cultivo de mayor riesgos a erosionar el suelo, como es el cultivo de yuca(Manihot suculenta) que predominó una erosión actual mayor a 12 t/ha/año, mientras que en el $73 \%$ del área se presenta erosión actual baja de 0.7 a 8.9 t/ha/año con predominio del cultivo con mayor cobertura como es el plátano y barbechos. La erosión actual estimada, es un problema de mediano riesgo.

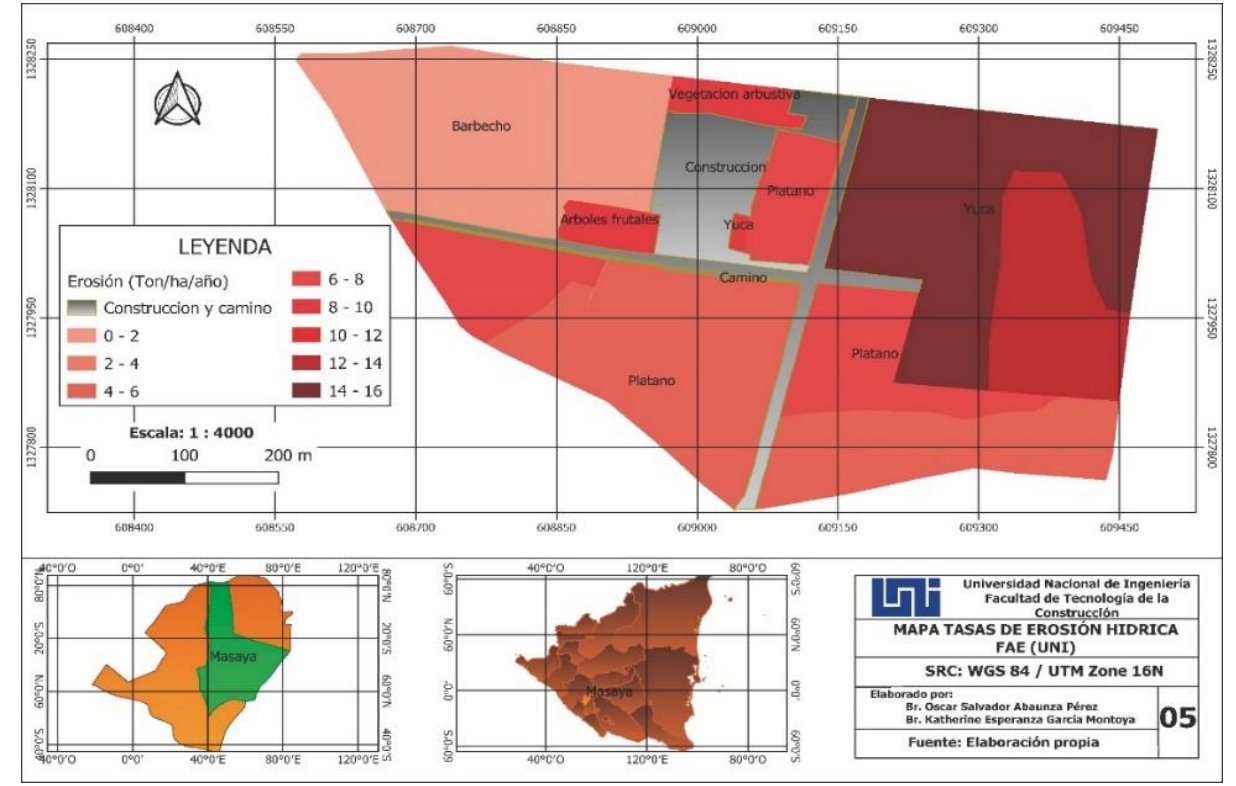

Figura 7: Mapa Tasa de erosión hídrica actual FAE (UNI) 


\section{Erosión hídrica potencial de los suelos de la FAE}

La Erosión Potencial (A) se estimó utilizando los primeros cuatro factores $(R, K, L, S)$, siendo el potencial erosivo inherente al sitio, es decir, las pérdidas de suelo que ocurrirán en ausencia de cualquier cobertura vegetal $(C)$ o Práctica de Conservación $(P)$, haciendo que las pérdidas potenciales se reduzcan con la presencia de estos dos últimos factores ver figura 8 , en las cuales pueden implementarse planes de conservación u otras medidas que mitiguen el efecto en los sitios donde la pérdida de suelo sea de moderada a alta.

La estimación de la erosión potencial en el área de estudio identificó zonas vulnerables, entre ellas, las establecidas con cultivo de plátano, indicando que, de no existir cobertura vegetal en esta zona, el grado de erosión sobrepasarían los $90 \mathrm{Ton} / \mathrm{Ha}$-año, en el caso de otros cultivos como la yuca las perdidas oscilarían entre 48 a 74 Ton/ha-año, tasas de erosión clasificadas como severas.

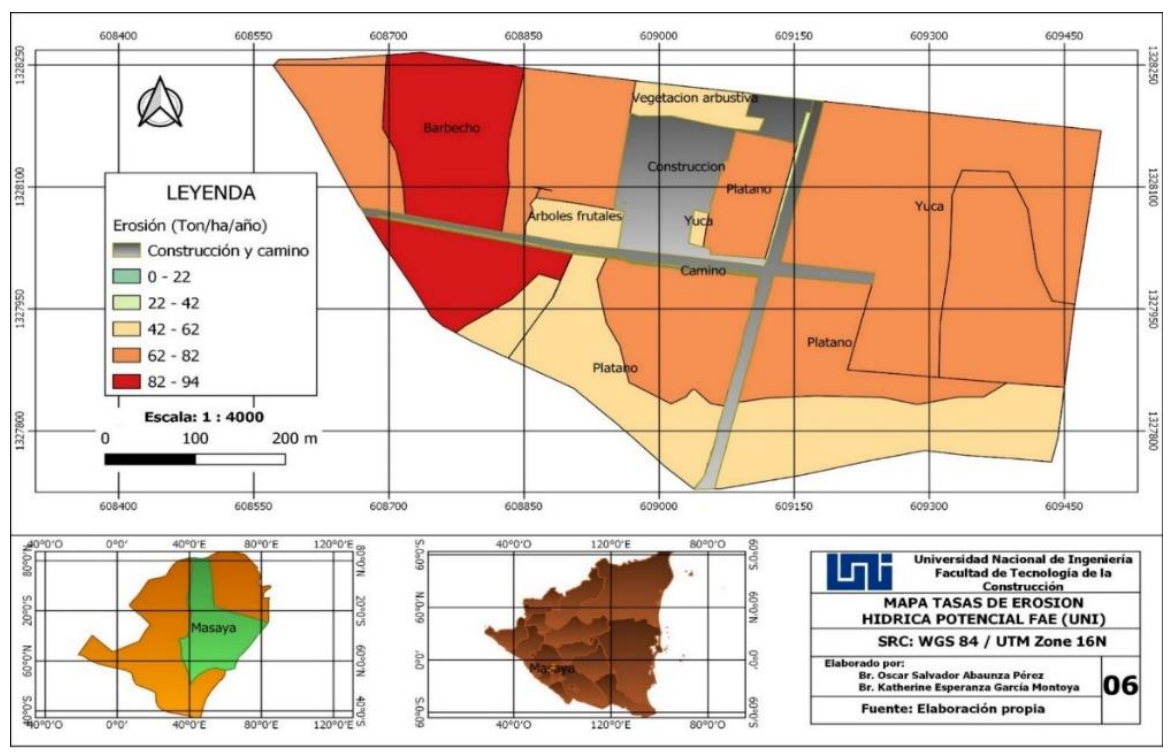

Figura 8: Mapa Tasas de erosión hídrica Potencial FAE(UNI)

\section{CONCLUSIONES}

De acuerdo las características físicas y químicas del suelo de la finca FAE/UNI, son suelos porosos, bien aireados y con buen drenaje, con densidad real de $2.14 \%$ clasificada como baja, Capacidad de Campo es mediana con $22.42 . \%$ capaz de retener el suelo luego de su saturación; la textura con $32.48 \%$ de arcilla, $63.12 \%$ de limo y $4.4 . \%$ de arena clasificada como textura franco arcillosa limosa y la materia orgánica fue $4,27 \%$ con índice de alto, caracterizado como buena calidad edáfica para cultivos agrícolas. El Factor de Erosividad (R) promedio para las tres estaciones meteorológica estudiada resultó ser de $6448.5 \mathrm{MJ} . \mathrm{mm} . h a^{-1}$, clasificada como una erosividad moderada producida por las lluvias moderadas. La determinación de la erodabilidad del suelo por medio de $\mathrm{K}$ teórico utilizando el nomograma de (Wischmeier \& Smith, 1971), arrojo un valor 0.06. indicando un rango bajo, es decir resistente al desprendimiento por la salpicadura o flujo laminar. El K experimental resulto ser de 0.05 clasificado como rango bajo, coincidiendo con el valor de $k$ teórico, en que ambos 
valores indican que el suelo presenta resistencia al desprendimiento por efecto de la lluvia. Los valores obtenidos de erosión hídrica actual están dentro de la categoría de Erosión baja (menor de 12 ton/ha/año), a excepción de los polígonos en donde está establecido el cultivo de yuca, donde las pérdidas son mayores a 12 ton/ha/año clasificada como media.

\section{AGRADECIMIENTOS}

A la Facultad de Tecnología de la Construcción (FTC) de la Universidad Nacional de Ingeniería a través de MSc José Méndez Úbeda jefe de Departamento de la carrera de Ingeniería Agrícola, por su apoyo logístico, a la Dra. Martha Orozco Izaguirre de la Universidad Nacional Agraria (UNA) por su apoyo técnico y al Ing. Zadis Vanegas por su apoyo y disposición durante los ensayos de campo y de laboratorio.

\section{REFERENCIAS}

Blanco M, C. (2019). Erosión hídrica en la microcuenca del cauce 31 de diciembre. NEXO, Revista cientifica, 12.

Blanco M, C. (2019). Erosión hídrica en la microcuenca del cauce 31 de diciembre. NEXO, Revista cientifica. Obtenido de https://doi.org/10.5377/nexo.v32i02.9261

Cerrato L, S. C., \& Téllez E, I. G. (2006). Caracterización fisico-quimico y morfológica para el uso y manejo de los suelos de la finca "La puebla" comunidad "Santa Clara" comarca "La cortezas" Municipio "Tisma", departamento de Masaya. Managua: Universidad Nacional de Ingenieria.

Espinales E. CATASTRO. (1971). Descripción de suelos, Levantamiento de suelos de la región Pacífica de Nicaragua. Managua: Catastro de inventario de recursos naturales de Nicaragua INETER.

Foster et al, G. D. (1977). A runoff erosivity factor and variable slope length exponents for soil loss estimates. Trans ASAE 20(4).

INETER, \& COSUDE. (2005). EROSION HIDRICA, mapas de amenazas. Managua, Nicaragua.

Monge R, \& Aguilar J. (2008). Diseño de un simulador de lluvia portátil con fines didácticos y de investigación para la cuantificación de la infiltración, escorrentia y erosión. Costa Rica.

Murillo G. (1990). Erodabilidad de cuatro series de suelos determinadas por el mini-simulador de Iluvia y el nomograma de Wischmeier. Managua.

Renard et al, K. F. (1997). Predicting Soil Erosion by Water: A Guide to Conservation Planning with the Revised Universal Soil Loss Ecuation (RUSLE). En U. D. Agriculture. Washington, D.C.: Agriculture Handbook No.703. 
Rivera C, \& Alguera E. (2016). Estimación del factor de erodabilidad (factor K) del suelo en el Centro Experimental Agrícola CEA-UNI, Municipio de Tisma, Departamento de Masaya. Managua, Nicaragua.

Santos A, P., \& Orrego E, L. (2016). Riesgo potencial a erosion hidrica para la planificación del manejo y conservación de suelos de la agroindustria azucarera gualtemateca. Guatemala.

Wischmeier \& Smith. (1976). A rainfall erosion index for universal soil Loss Equation, Soil Science Society Proc. USA.

Wischmeier y Smith, 1., Roffe Ligtenberg et al, 2., \& FAO. (1985). Tabla de niveles de intensidad de erosión o pérdida de suelo adaptados de criterios internacionales.

Wischmeier, \& Smith. (1971). Predicting Rainfall Erosion Losses - A Guide to Conservation Planning. Washington, D.C.: U.S.: Agriculture Handbook, 537. Department of Agriculture (USDA).

\section{SEMBLANZA DE AUTORES}

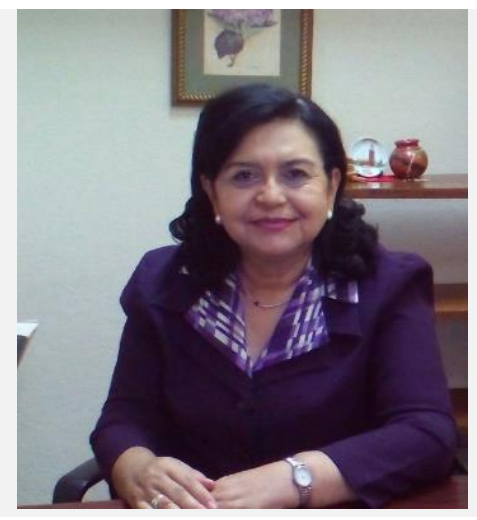

Rosario Verónica Sotelo Contreras: Rosario Verónica Sotelo Contreras, Ingeniera agrónoma, con Maestría en Ordenación rural en función del medio ambiente, además es investigadora y docente universitario por más de 30 años.

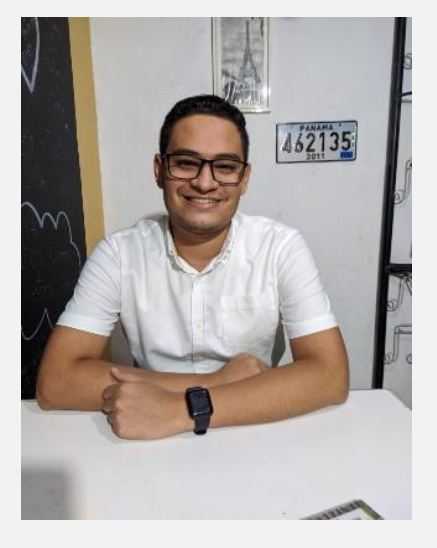

Oscar Salvador Abaunza Pérez: Ingeniero agrícola, con conocimientos en Sistemas de Información Geográfica (SIG), así como en manejo de gestores de base de datos, específicamente Transact-SQL con Microsoft SQL Server. Actualmente se desempeña como analista climático y en la elaboración de boletines agroclimáticos para los cultivos de cacao, maní y maíz para diferentes cooperativas del país. 


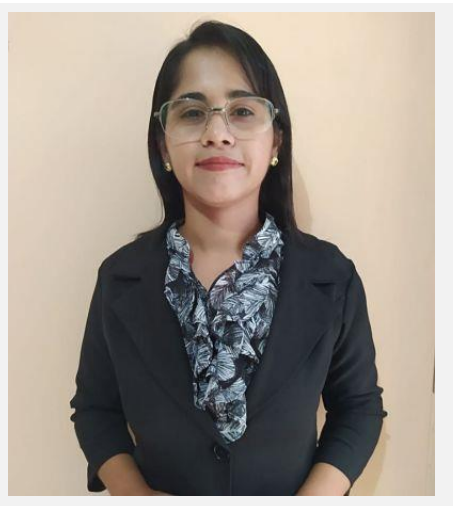

Katherine Esperanza García Montoya: Ingeniero agrícola, miembro activo de IEEE Sección Nicaragua en el capítulo de EDS-SIGHT, formó parte del programa IBT-200 de GRID ALTERNATIVE, con conocimientos en suelo y energía solar. Actualmente se desempeña como Ingeniera de ventas y proyectos en el área de energía solar.

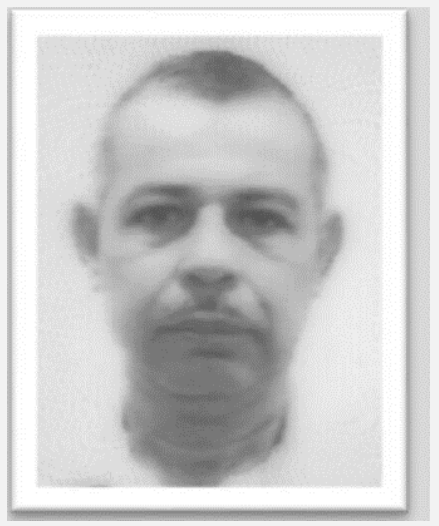

Miguel Enrique Blanco Chávez: Ingeniero Civil, con maestría en Ingeniería Ambiental. Trabaja en el Programa de Investigación de Estudios Nacionales y Servicios del Ambiente (PIENSA) de la UNI. Es docente de Hidráulica e Hidrología en la Facultad Tecnología de la Construcción más de 20 años. Actualmente imparte clases en la Maestría en Ingeniería Ambiental de la UNI. Es consultor en Hidrología e Hidráulica. 